\title{
A thermographic and clinical comparison of three intra-articular steroid preparations in rheumatoid arthritis
}

\author{
H. A. BIRD, E. F. J. RING, AND P. A. BACON
}

From the Royal National Hospital for Rheumatic Diseases, Bath

SUMMARY We have compared three intra-articular steroid preparations in a double blind study orio 30 patients with rheumatoid arthritis and bilateral synovitis of the knees. One knee was injected with $1.0 \mathrm{ml}$ of either prednisolone t-butyl acetate, methyl prednisolone acetate, or triamcinolone hexacetonide, and the patients were followed up for 6 weeks with regular clinical and thermographic assessments. Thermographic improvement was seen with all 3 drugs but was greatest initially and longest lasting with triamcinolone. No significant systemic improvement was seen with any drug after a single injection, though all 3 steroid preparations suppressed endogenous cortisol.

Intra-articular steroid injections have been used in rheumatoid arthritis since 1951 when Hollander et al. (1951) first used hydrocortisone. Prednisolone followed (Rothermich and Phillips, 1957), and more recently both methyl prednisolone and triamcinolone have become available. In spite of the wide variety of proprietary brands there have been few comparative studies to advise on choice. Baine et al. (1967) found significantly greater improvement with methyl prednisolone than with prednisolone, and Dixon et al. (1972) found that triamcinolone produced marginally greater benefit with fewer local side effects than prednisolone acetate, but neither of these studies used an objective assessment of inflammation.

A previous study in this unit (Esselinckx et al., 1978) compared 3 intra-articular analogues of prednisolone (prednisolone acetate, prednisolone pivalate, and prednisolone t-butyl acetate) at 2 dosage levels, using infrared quantitative thermography to measure inflammation. Prednisolone tbutyl acetate produced a significantly greater and more sustained anti-inflammatory effect than the other 2 compounds. This was not significantly improved by increasing the dose from $50 \mathrm{mg}$ to 100 $\mathrm{mg}$, and since the higher dose caused more suppression of endogenous cortisol the lower dose was judged the better one.

We therefore decided to compare prednisolone

Accepted for publication 15 March 1978.

Correspondence to Dr H. A. Bird, Rheumatism Research Unit, University of Leeds School of Medicine, 36 Clarendon Road, Leeds LS2 9PJ. t-butyl acetate with 2 other synthetic steroid pre $\vec{\oplus}$ parations that on pharmacological grounds might be expected to be more efficacious than prednisolone. These were methyl prednisolone acetate and triams cinolone hexacetonide. We limited the comparisono to intra-articular injections of 1 knee in patients witlo classical or definite rheumatoid arthritis (ARA $\mathbb{D}$ criteria) and used the lowest dose recommended b $\overrightarrow{5}$ the manufacturers for this joint and disease. The study was double blind, and thermography providece an objective assessment of inflammation. Patients. were followed up for 6 weeks, and serial plasma cortisol levels were performed.

Many authors have suggested that a single intra응 articular injection may produce systemic improve ment. We therefore performed serial clinical assess ments of all joints and followed the full blood count and plasma viscosity throughout the study.

\section{Patients and methods}

Thirty outpatients or inpatients with classical of definite rheumatoid arthritis (ARA criteria) wer尺 allocated at random to 3 groups. Patients taking oral steroid preparations or immunosuppressive drugs were excluded, but patients receiving gold of D-penicillamine were included if dosage had beens stable for 3 months. Patients who had received intra articular or systemic steroids in the previous 30 months were excluded, and a prerequisite for entr. was that the disease affected both knees symmetri? cally. Drug therapy remained constant throughou the study. 


\section{STEROID PREPARATIONS}

A single intra-articular injection was given to $1 \mathrm{knee}$, chosen at random, without knowledge of the preparation. Synovial fluid was aspirated prior to injection but the joint was not then reaspirated. The dosages used were prednisolone t-butyl acetate (Codelcortone-TBA), $20 \mathrm{mg}$ in $1 \mathrm{ml}, 10$ patients; methyl prednisolone acetate (Depo-Medrone), 40 $\mathrm{mg}$ in $1 \mathrm{ml}, 10$ patients; and triamcinolone hexacetonide, (Lederspan), $20 \mathrm{mg}$ in $1 \mathrm{ml}, 10$ patients. The steroid preparations were undiluted and not mixed with local anaesthetic.

\section{ASSESSMENTS}

Patients attended a special clinic on days $0,2,4,7$, 14, 28, and 42. Thermograms of both knees were carried out before injection on day 0 and at the same time of day on each subsequent visit under standardised conditions as described by Ring (1975). The thermographic index (TI) of the injected and noninjected knee was recorded by the method of Collins et al. (1974) and the change in TI from the preinjection readings calculated. Articular index (Ritchie et al., 1968), grip strength, global pain score on a visual analogue scale, and duration of morning stiffness were recorded on days $0,7,14$, 28 , and 42 .

\section{INVESTIGATIONS}

Full blood count and plasma viscosity were performed on days 0,14 , and $42.10 \mathrm{ml}$ of venous blood for cortisol estimation was collected into heparinised tubes on days $0,2,4,7,14$, and 28 . Plasma was then separated and stored at $-20^{\circ} \mathrm{C}$ before estimation.

Cortisol estimations were performed by the fluorimetric method of Mattingly (1962). The cortisol was extracted from plasma by mixing $2.0 \mathrm{ml}$ with 15.0 $\mathrm{ml}$ dichloromethane and centrifuging the mixture at $2000 \mathrm{rpm}$ for 10 minutes at $18^{\circ} \mathrm{C}$. The dichloromethane extract was decanted and $10.0 \mathrm{ml}$ transferred to a smaller tube. The fluorescence reagent was prepared by mixing $70 \mathrm{ml}$ concentrated sulphuric acid with $30 \mathrm{ml}$ ethyl alcohol. The cortisol extract was shaken vigorously with $5.0 \mathrm{ml}$ of fluorescence reagent for 20 seconds and the supernatant dichloromethane pipetted off. The acid extract was then transferred to a fluorimetry cell and fluorescence at $525 \mathrm{~nm}$ read thirteen minutes after mixing using an exciting light of $472 \mathrm{~nm}$ wavelength. The results were calibrated with a reagent blank and compared to a series of standard cortisol solutions estimated by the same method. Cortisol levels were then subtracted from the preinjection level.

Student's $t$ test was used throughout the study.

\section{Results}

\section{CLINICAL ASSESSMENTS}

There were no significant differences between any of the 3 groups for any parameter before the injection. The articular index showed no significant improvement in any group, the most improvement being obtained with methyl prednisolone at 14 days $(\mathbf{P}=0 \cdot 1)$. Morning stiffness also showed no significant improvement in any group, triamcinolone at 7 days $(0.5>\mathbf{P}>0.1)$ being best. For pain score there was a significant improvement in the triamcinolone group at 7 days $(0.05>P>0.02)$, though this was not maintained at 14 days $(0.5>P>0 \cdot 1)$. No other group showed improvement. Grip strength showed no significant changes in any group, the greatest improvement being with triamcinolone at 28 days $(P>0 \cdot 5)$. Differences between groups were not significant at any time.

\section{LABORATORY ASSESSMENTS}

The 3 groups were intially well matched with respect to full blood count and plasma viscosity. No significant changes were seen in haemoglobin or white blood count at any time, either within or between groups. Plasma viscosity fell slightly by 6 weeks in all 3 groups, the fall being greatest with prednisolone t-butylacetate, though this was not significant $(0.5>$ $\mathbf{P}>\mathbf{0} \cdot \mathbf{1}$ ).

\section{THERMOGRA PHIC ASSESSMENTS}

The improvement in injected knees for the 3 groups is shown in Fig. 1. All groups improved with the greatest change at 1 week. Improvement was subsequently lost, though knees injected with triamcinolone maintained their improvement at 6 weeks, when knees injected with the other 2 preparations had returned to their preinjection value. Moreover, the improvement with triamcinolone was more pronounced than with the other preparations. As to change within groups, the triamcinolone improvement was highly significant at 7 days $(\mathrm{P}<0.001)$ and significant at 4,14 , and 28 days. Change with methyl prednisolone was significant at 2 days $(0.02>$ $\mathbf{P}>0.01$ ) and 4 days; change with prednisolone t-butyl acetate significant only at 4 days $(0.02>P>0.01)$.

The improvement in the contralateral non-injected knees is shown in Fig. 2. Although the knees of patients on methyl prednisolone and prednisolone t-butyl acetate showed no change, the non-injected knees of those on triamcinolone showed an improvement of similar magnitude to the knees injected with methyl prednisolone, though this improvement did not reach significant levels.

\section{PLASMA CORTISOL ESTIMATIONS}

The fall in endogenous plasma cortisol in the 3 


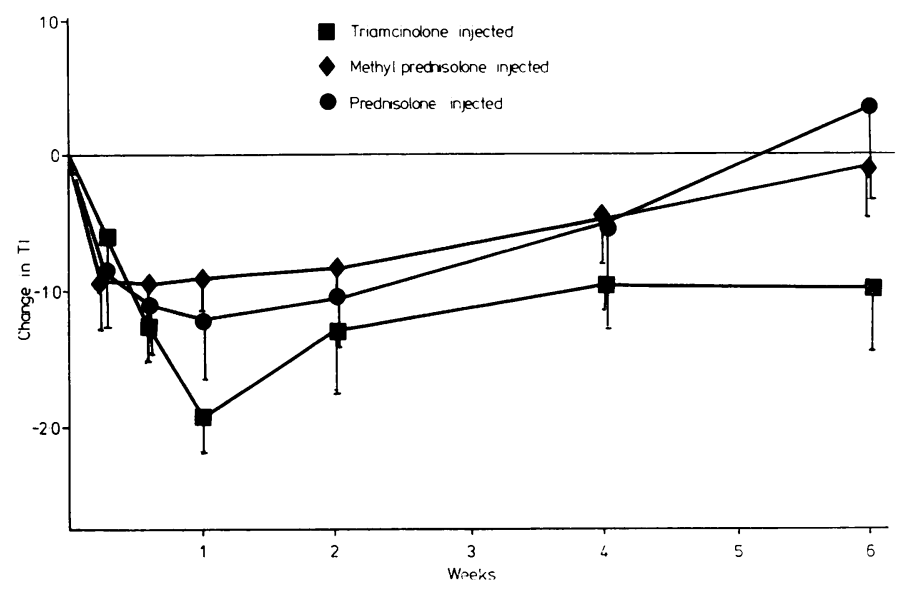

Fig. 1 Mean fall in thermographic indee $\mathbb{\mathbb { \Omega }}$ (TI) of injected knees from groups of patients treated with prednisolone $t$ butyl acetate, methyl prednisolone acetate, and triamcinolone hexacetonide Bars represent standard error of the mean.

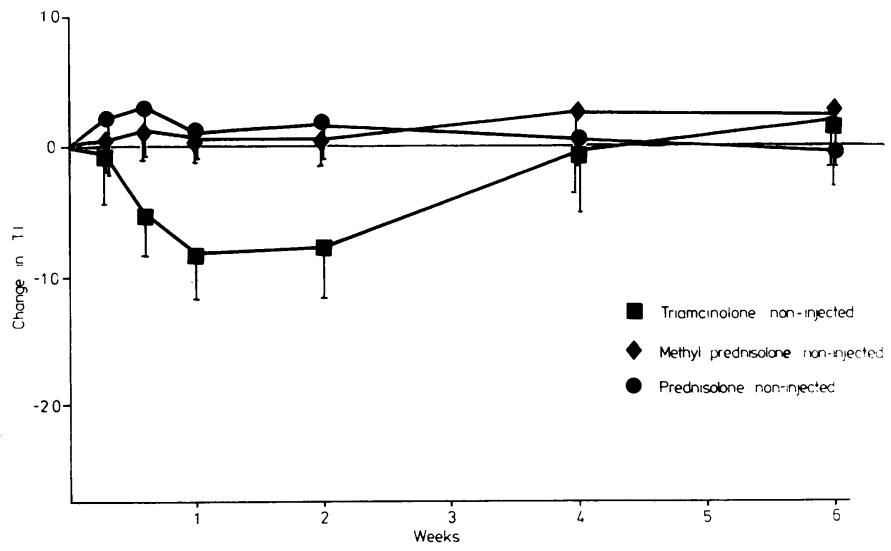

Fig. 2 Mean change in thermographic index of the non-injected knees from groups of patients treated as in Fig. 1. Bars represent standard error of the mean.

groups is shown in Fig. 3. Adrenal suppression occurred with all 3 drugs, being maximal by 2 or 4 days and being most pronounced in those patients receiving prednisolone t-butyl acetate.

\section{Discussion}

Although many patients showed modest clinical improvement after intra-articular injection, we found no significant systemic improvement with any of the steroid preparations used. A controlled study would be required to ascertain whether the minor changes seen resulted from steroid therapy or whether they arose because of regular clinical follow-up.

Thermography showed clear differences between groups of injected knees. Improvement occurred with all 3 drugs but the pattern seen with triamcinolone differed in 2 respects: the initial maximum improvement was greater, and the improvement was maintained to the end of the study at 6 weeks, stage when the other groups had reverted to normaR Improvement with triamcinolone reached a greates level of significance than with the other 2 drugs.

Thermography also revealed differences betwee groups of non-injected knees, there being an imr provement in the triamcinolone group not seen with the other 2 steroid preparations. The improvemen was comparable in magnitude to the injected kneeș of the methyl prednisolone group.

It is not clear why injected and non-injected knees behaved differently according to the steroid pre paration used. The injected knee was chosen at random and there was no significant initial differenced between knees (mean TI injected knees/non-injecte $\mathbb{P}$ knees $=4 \cdot 71 / 4 \cdot 09$ ). When exogenous plasma steroi $\$$ levels are measured (Esselinckx et al., 1978) it can bS shown that prednisolone analogues enter the syso temic circulation from the joint very quicklyo 


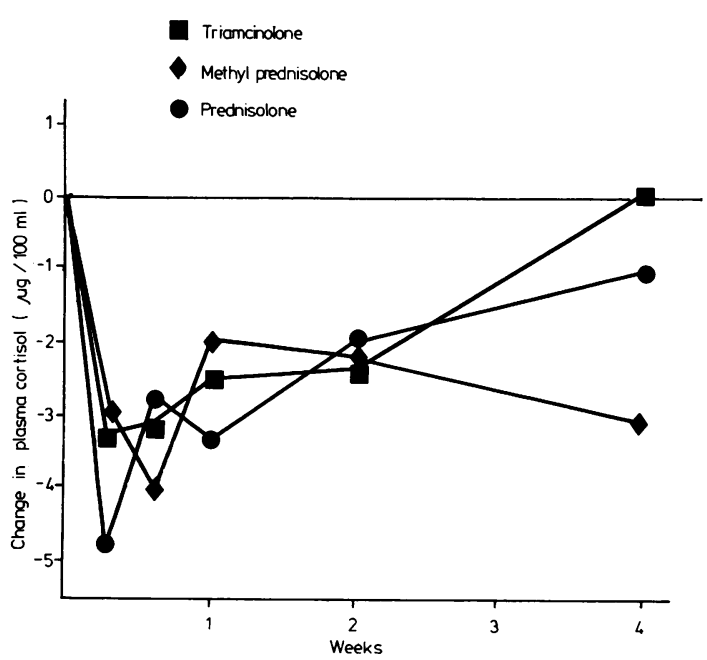

Fig. 3 Mean fall in endogenous plasma cortisol for the 3 groups of patients treated as in Fig. 1. (Conversion to $S I: 1 \mu \mathrm{g} / 100 \mathrm{ml}=27.6 \mathrm{nmol} / \mathrm{l}$.)

With methotrexate, a drug for which adequate and sensitive bioassays exist, the drug is always present in synovial fluid of 1 knee half an hour after injection into the synovial cavity of the opposite knee (Bird et al., 1977). It is therefore possible that improvement of the non-injected knee in patients injected with triamcinolone represents a systemic effect of this drug, even though this was not reflected in the global clinical assessments. One possible explanation for this is that the patients were selected because of bilateral knee synovitis and their other joints were less active. Why methyl prednisolone and prednisolone t-butyl acetate did not also show this systemic effect is uncertain, and further studies using direct assays of these drugs are required to see if they leave the joint at different rates. Esselinckx et al. (1978) have suggested that systemic effect is a function of steroid solubility. In their comparison of 3 prednisolone analogues they found the least change in the contralateral knee of patients injected with prednisolone t-butyl acetate, the least soluble of the 3 prednisolone salts.

In this study the plasma cortisols do not help to elucidate this. They show adrenal suppression with all 3 drugs, initially most marked with prednisolone t-butyl acetate and longest lasting with methyl prednisolone. However, it is still possible that triamcinolone is more soluble than the other 2 drugs, since it may have caused a large early cortisol suppression not seen in this study, in which cortisol estimations were not performed in the first 24 hours after injection. This might explain its significant action in the non-injected knee.

On the basis of the lack of cortisol suppression at 4 weeks and its longer duration of action, triamcinolone appears to have advantages over the other 2 drugs in rheumatoid synovitis in the dosages that we tested.

We thank Dr J. A. Cosh and Dr A. St. J. Dixon for permission to include their patients in this study, Mr J. R. Lowe and Mr. J. S. Dixon for technical assistance, and Mrs J. Battersby for typing the script. The study was made possible through grants from Lederle Laboratories for thermographic estimations and from Merck Sharp and Dohme for cortisol estimations.

\section{References}

Baine, L. S., Balch, H. W., and Jacomb, R. G. (1967). Parental administration of 6 a Methyl Prednisolone 21 Acetate. Annals of Physical Medicine, 9, 43-54.

Bird, H. A., Ring, E. F. J., Daniel, R., and Bacon, P. A. (1977). Comparison on intra-articular methotrexate with intra-articular triamcinolone hexacetonide by thermography. Current Medical Research and Opinion, 5, 151146.

Collins, A. J., Ring, E. F. J., Cosh, J. A., and Bacon, P. A. (1974). Quantitation of thermography in arthritis using multi-isothermal analysis. 1. The Thermographic Index. Annals of the Rheumatic Diseases, 33, 113-115.

Dixon, A. St. J., Cosh, J. A., and Kersley, G. D. (1972). Local corticosteroid therapy for painful rheumatic states. A comparison of triamcinolone hexacetonide (Lederspan) and Prednisolone Acetate (Precortisyl). Clinical Trials Journal, 3, 14-18.

Esselinckx, W., Bacon, P. A., Ring, E. F. J., Crooke, D., Collins, A. J., and Demottaz, D. (1978). A thermographic assessment of three intra-articular prednisolone analogues given in rheumatoid synovitis. British Journal of Clinical Pharmacology, 5, 447-451.

Hollander, J. L., Brown, E. M., Jessar, R. A., and Brown, C. Y. (1951). Comparative effects of use of hydrocortisone as a local anti-arthritic agent. Journal of American Medical Association, 147, 1629-1635.

Mattingly, D. (1962). A simple fluorimetric method for the estimation of free 11-hydroxycorticosteroids in human plasma. Journal of Clinical Pathology, 15, 374-379.

Ring, E. F. J. (1975). Thermography and rheumatic diseases. Bibliotheca Radiologica, 6, 97-106.

Ritchie, D. M., Boyle, J. A., McInnes, J. M., Jasani, M. K. Dalakos, T. G., Grieveson, P., and Buchanan, W. W. (1968). Clinical studies with an articular index for the assessment of joint tenderness in patients with rheumatoid arthritis. Quarterly Journal of Medicine, 147, 393-406.

Rothermich, N. O., and Phillips, U. K. (1957). Local injection of Prednisolone TBA in the treatment of rheumatic diseases. Ohio State Medical Journal, 53, 43-46. 